\title{
Objectivity tests for Navier-Stokes simulations: The revealing of non-physical solutions produced by Laplace formulations
}

\author{
Alejandro C. Limache ${ }^{\mathrm{a}, *, 1}$, P.J. Sánchez ${ }^{\mathrm{a}}$, Lisandro D. Dalcín ${ }^{\mathrm{a}}$, Sergio R. Idelsohn ${ }^{\mathrm{b}, 2}$ \\ a International Center of Computational Methods in Engineering (CIMEC), INTEC-CONICET-UNL, Guemes 3450, Santa Fe 3000, Argentina \\ ${ }^{\mathrm{b}}$ International Center for Numerical Methods in Engineering (CIMNE), Barcelona, Spain
}

\section{A R T I C L E I N F O}

\section{Article history:}

Received 5 August 2007

Received in revised form 24 March 2008

Accepted 15 April 2008

Available online 6 May 2008

\section{Keywords:}

Navier-Stokes equations

Objectivity

Laplace diffusion operator

Annular cavity

Free-surfaces

Boundary conditions

\begin{abstract}
A B S T R A C T
Laplace formulations are weak formulations of the Navier-Stokes equations commonly used in computational fluid dynamics. In these schemes, the viscous terms are given as a function of the Laplace diffusion operator only. Despite their popularity, recently, it has been proven that they violate a fundamental principle of continuum mechanics, the principle of objectivity. It is remarkable that such flaw has not being noticed before, neither detected in numerical experiments. In this work, a series of objectivity tests have been designed with the purpose of revealing such problem in real numerical experiments. Through the tests it is shown how, for slip boundaries or free-surfaces, Laplace formulations generate non-physical solutions which widely depart from the real fluid dynamics. These tests can be easily reproduced, not requiring complex simulation tools. Furthermore, they can be used as benchmarks to check consistency of developed or commercial software. The article is closed with a discussion of the mathematical aspects involved, including the issues of boundary conditions and objectivity.
\end{abstract}

(c) 2008 Elsevier B.V. All rights reserved.

\section{Introduction}

The Navier-Stokes (NS) equations are extensively used in fluid mechanics. They are typically presented, as partial differential equations (PDE), in the following format:

$\rho \frac{\partial \boldsymbol{v}}{\partial t}+\rho \nabla \boldsymbol{v} \cdot \boldsymbol{v}+\nabla p-\mu \nabla^{2} \boldsymbol{v}=0$

$\nabla \cdot \boldsymbol{v}=0$,

where $\rho$ is the density, $p$ the static pressure, $\boldsymbol{v}$ the velocity vector and $\mu$ the fluid viscosity. Such traditional PDE format is called the Laplace format because the viscous terms appear simply in the form of the Laplace diffusion operator $\nabla^{2}$ applied to the velocity vector $\boldsymbol{v}$.

One important aspect of the Laplace diffusion operator is that it does not couple velocity components, as can be seen from its mathematical expression:

$$
\nabla^{2} \boldsymbol{v}=\left[\begin{array}{c}
\nabla^{2} u \\
\nabla^{2} v \\
\nabla^{2} w
\end{array}\right]
$$

\footnotetext{
* Corresponding author. Tel.: +54 342 155439825; fax: +54 3424511169 .

E-mail addresses: alejandrolimache@hotmail.com, alimache@santafe-conicet. gov.ar (A.C. Limache)

URL: http://www.cimec.org.ar/alimache (A.C. Limache).

1 Researcher of CONICET.

2 ICREA Research Professor.
}

On the other hand, it is clear from Eq. (1) that the pressure and the convective terms do produce a coupling between velocity components. One naturally expects these features to be also present when solving numerically the Navier-Stokes equations.

In the case of modern numerical schemes based on integral forms (also called weak forms), there are two common approaches: either to use Laplace formulations, or to use Divergence formulations (see for example, $[3,8,9,19,22,23,30,34,44-46,48])$. Laplace formulations have been traditionally derived from the Laplace format given in Eqs. (1) and (2). In them, like in such PDE counterpart, the viscous terms depend only on the Laplace diffusion operator:

$\mu \llbracket \boldsymbol{v}=$ weak form $\left[\mu \nabla^{2} \boldsymbol{v}\right]$.

On the other hand, Divergence formulations are derived from the Divergence format of the Navier-Stokes equations (see Eqs. (6) and (7) below).

Despite the common use of both formulations, recently, Limache and Idelsohn [20] have shown that Laplace formulations violate a main axiom of continuum mechanics: the principle of objectivity (see [27,15]). In a following article [21], the proof was extended to cover also two-phase flows (i.e. discontinuities and the so called level set methods $[22,48])$. They also presented the first numerical examples showing the spurious effects of this violation. The examples required the use of the particle finite element method PFEM [16,17].

As it will be shown, the failure of Laplace formulations is caused by an over simplification of the boundary conditions. Simplificative 
considerations are standard practice in physics. However, in this case, such unaccurate (but historically accepted) boundary treatment leads to an unforeseen consequence: the violation of a fundamental principle of continuum mechanics. Indeed, the detection of the failure and the understanding of the nature of the error did not come from noticing any problem on boundary conditions but came from noticing Objectivity issues. Objectivity played a key role in the discovery of the problem. First, when some numerical simulations showed a strange incapacity of pseudo-fluids (hypo-elastic) materials to achieve rotational motions. Second, when comparisons with the Divergence formulation, which is clearly objective, showed not a boundary condition problem but a volumetric term missed. This eventually lead to the demonstration that the error, made in Laplace formulations, was not simply another boundary condition approximation but, also, an error equivalent to the simulation of a non-objective fluid material. Furthermore, in order to emphasize the importance of objectivity, it should be mentioned that the tests presented in this article have been devised not by looking at boundary conditions but by trying to detect objectivity failures.

It is remarkable indeed that such objectivity flaw has not being noticed before, neither detected in numerical experiments, especially considering that Laplace formulations have been in use for several decades. The reason for this situation is probably because in many practical problems those formulations just give the correct physical response. The point made in this paper is that, surprisingly, in other circumstances they just do not. In the present work, it will be shown how a series of carefully devised numerical exper-

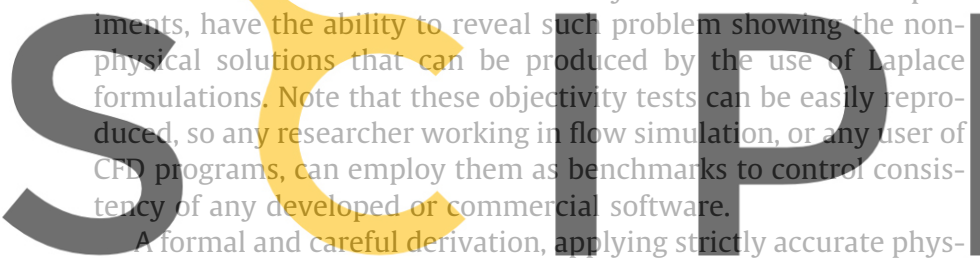
ical boundary conditions, shows that the source of the problem lies in that Laplace formulations have a missing integral boundary

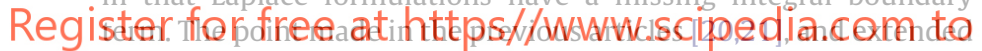
here to the case of slip boundary conditions, is that this missing term cannot be neglected and actually is extremely important. If one does take it into account, one arrives to a corrected Laplace formulation which is objective and, as a consequence, consistent. Unfortunately, the correction links velocity components and couples more strongly the momentum equations. Furthermore, the viscous terms cannot longer be written as a function of the Laplace diffusion operator only.

The article is structured in the following way. First, the Divergence format and the Laplace format, i.e., the two most common PDE formats of the Navier-Stokes equations are introduced. Then from these two formats, the two most commonly used weak formulations, the Divergence formulation and the Laplace formulation, are derived. Next, a series of simple flow experiments will be presented and simulated using the finite element method (FEM). The purpose of these tests is to evaluate if there are any differences between solutions obtained using either Divergence formulations or Laplace formulations. It will be seen that in many classical tests both approaches give the same results, as most people would expect. However, it will also be seen that, for the flow examples named objectivity tests, while the Divergence approach still produces correct physical answers, the Laplace scheme widely departs from the correct solution, producing non-physical results. The article is closed with a theoretical discussion explaining, from a mathematical point of view, many aspects observed in the numerical experiments, including the role of objectivity. In that section, the corrected Laplace formulation will be also presented, showing how existing code based on Laplace schemes can be fixed by adding an appropriate boundary correction term.

\section{Navier-Stokes equations}

The Navier-Stokes equations describe the dynamics of incompressible newtonian fluids. Such fluids are defined through their constitutive equation:

$\boldsymbol{\sigma}=-p \boldsymbol{I}+\mu \nabla \boldsymbol{v}+\mu(\nabla \boldsymbol{v})^{\mathrm{T}}$,

where $\boldsymbol{\sigma}$ is the Cauchy stress tensor and $\boldsymbol{I}$ the second order identity tensor. The so called Divergence format of the Navier-Stokes equations is obtained by inserting (5) into the equations of conservation of mass and momentum of continuum mechanics:

$\rho \frac{\partial \boldsymbol{v}}{\partial t}+\rho(\nabla \boldsymbol{v}) \cdot \boldsymbol{v}-\nabla \cdot\left(-p \boldsymbol{I}+\mu \nabla \boldsymbol{v}+\mu(\nabla \boldsymbol{v})^{\mathrm{T}}\right)=0$

$\nabla \cdot \boldsymbol{v}=0$

One can recover the Navier-Stokes equations in the Laplace format, defined in Eqs. (1) and (2), by expanding the terms containing the Divergence operator in Eq. (6):

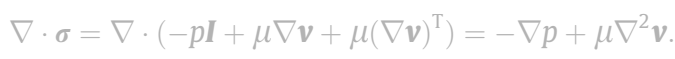

Note that the term $(\nabla \boldsymbol{v})^{\mathrm{T}}$ produces no contribution because:

$\nabla \cdot(\nabla \boldsymbol{v})^{\mathrm{T}}=\nabla(\nabla \cdot \boldsymbol{v})=0$

for incompressible flows. The Laplace format and the Divergence format are of course equivalent, they are just different ways of writ-
ing the same PDE (under the assumption of constant yiscosity).
As the fluid moves in a domain $\Omega$, the NS equations (written
either in Divergence or Laplace formats) have to be solved subject
to appropriate physical boundary conditions, prescribing either the
velocity or external surface forces (tractions) at the boundary sur-
faces $I$ of $\Omega$. Then, one can account for three possible types of
boundary conditions: no-slip, slip, and free-surface conditions. For simplicity and clarity, it will be assumed here that the traction

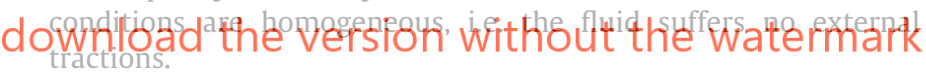

\subsection{No-slip boundary conditions}

At no-slip boundaries $\Gamma_{\text {no-slip, all the flow velocity components }}$ are imposed:

$\boldsymbol{v}=\overline{\boldsymbol{v}} \quad$ on $\quad \Gamma_{\text {no-slip }}$

\subsection{Slip boundary conditions}

At slip boundaries $\Gamma_{\text {slip }}$, the normal velocity component $v_{n}$ vanishes and there are not tangential stresses. This means:

$$
\left.\begin{array}{l}
v_{n}=\boldsymbol{v} \cdot \boldsymbol{n}=0 \\
(\boldsymbol{\sigma} \cdot \boldsymbol{n}) \cdot \boldsymbol{t}=0 \quad \forall \boldsymbol{t} \in\left\{\boldsymbol{n}^{\perp}\right\}
\end{array}\right\} \quad \text { on } \quad \Gamma_{\text {slip }},
$$

where $\boldsymbol{n}$ is the unit vector normal to the boundary surface $\Gamma$ and $\boldsymbol{t}$ is any of the unit vectors tangent to the surface.

\subsection{Free-surface boundary conditions}

At free-surfaces $\Gamma_{\mathrm{fs}}$, the velocity components are let free and zero tractions are imposed:

$\boldsymbol{\sigma} \cdot \boldsymbol{n}=0 \quad$ on $\quad \Gamma_{\mathrm{fs}}$.

or equivalently:

$$
\left.\begin{array}{l}
(\boldsymbol{\sigma} \cdot \boldsymbol{n}) \cdot \boldsymbol{n}=0 \\
(\boldsymbol{\sigma} \cdot \boldsymbol{n}) \cdot \boldsymbol{t}=0 \quad \forall \boldsymbol{t} \in\left\{\boldsymbol{n}^{\perp}\right\}
\end{array}\right\} \quad \text { on } \quad \Gamma_{\mathrm{fs}} .
$$


Remark 1. A normal traction condition must exist in order to fix the pressure, then for the cases of domains with no free-surfaces, the internal pressure $p$ must be fixed at some point $\boldsymbol{x}_{0}$ on the surface, i.e., for such cases the condition $p=0$ for $\boldsymbol{x}_{0} \in \Gamma$ will be used.

Next, the two most commonly used weak/variational formulations will be derived using a FEM-like approach. The first one will be obtained (using classical arguments) from the Laplace format (1) and (2) and will lead to the Laplace formulation. The second will be derived starting from the Divergence format (6) and (7) leading to the Divergence formulation. After presenting them in Sections $3-5$, their behavior will be compared via a series of simple flow tests in Section 6.

\section{Laplace formulations for fully-imposed domains}

Laplace formulations are weak formulations of the NS equations initially derived for applications dealing with flows in fully-imposed domains, i.e., when $\Gamma=\Gamma_{\text {no-slip. }}$ They can be obtained in a straightforward manner from the Laplace format Eqs. (1) and (2), as shown below. Integration of the scalar product of Eq. (1) with a vector $\boldsymbol{w}$ of admissible weighting functions over the fluid domain $\Omega$ yields:

$\boldsymbol{w} \cdot \rho\left[\frac{\partial \boldsymbol{v}}{\partial t}+\nabla \boldsymbol{v} \cdot \boldsymbol{v}\right] \mathrm{d} \Omega+\int_{\Omega} \boldsymbol{w} \cdot \nabla p \mathrm{~d} \Omega-\int_{\Omega} \boldsymbol{w} \cdot \mu \nabla^{2} \boldsymbol{v} \mathrm{d} \Omega=0$

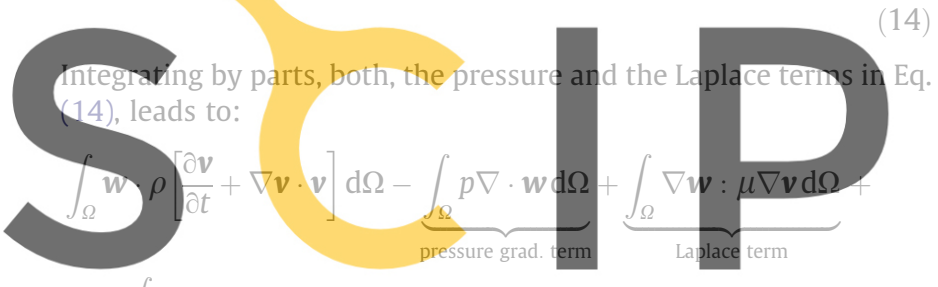

$\boldsymbol{w} \cdot(-p \boldsymbol{I}+\mu \nabla \boldsymbol{v}) \cdot \boldsymbol{n} \mathrm{d} \Gamma=0$

(15)

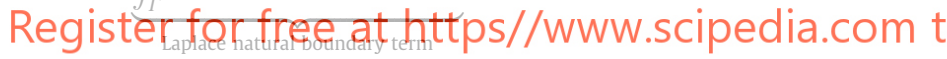

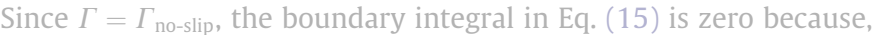
in such a case, $w$ vanishes everywhere on the boundary. Using this property and performing a similar variational procedure on the continuity Eq. (2) with an admissible weighting function $q$, one gets the general expression defining Laplace formulations:

$\underbrace{\int_{\Omega} \boldsymbol{w} \cdot \rho\left[\frac{\partial \boldsymbol{v}}{\partial t}+\nabla \boldsymbol{v} \cdot \boldsymbol{v}\right] \mathrm{d} \Omega}_{\mathbb{M} \frac{\mathrm{d} \boldsymbol{v}}{\mathrm{d} t} \mathbb{C}(\boldsymbol{v})}-\underbrace{\int_{\Omega} p \nabla \cdot \boldsymbol{w} \mathrm{d} \Omega}_{-\mathbb{G} p}+\underbrace{\int_{\Omega} \nabla \boldsymbol{w}: \mu \nabla \boldsymbol{v} \mathrm{d} \Omega}_{-\mu \llbracket \boldsymbol{v}}=0$,

$\underbrace{\int_{\Omega} q \nabla \cdot \boldsymbol{v} \mathrm{d} \Omega}_{\mathbb{D} \boldsymbol{v}}=0$

Using the symbolic operators defined below the underbraces in Eqs. (16) and (17), one can write the Laplace formulation simply as:

$\mathbb{M}\left(\frac{\mathrm{d} \boldsymbol{v}}{\mathrm{d} t}\right)+\mathbb{C}(\boldsymbol{v})+\mathbb{G}(p)-\mu \llbracket(\boldsymbol{v})=0$,

$\mathbb{D}(\boldsymbol{v})=0$.

Note that the Laplace diffusion operator term has been denoted with the symbol $\mu \llbracket$.

\section{Divergence formulation for fully-imposed domains}

To get the Divergence formulation for the case of fully-imposed domains ( $\Gamma=\Gamma_{\text {no-slip }}$ ) we start from the Divergence format (6) and
(7) instead of the Laplace format. Integrating the scalar product of Eq. (6) with a vector $\boldsymbol{w}$ of admissible weighting functions over the fluid domain $\Omega$ one gets:

$\int_{\Omega} \boldsymbol{w} \cdot \rho\left[\frac{\partial \boldsymbol{v}}{\partial t}+\nabla \boldsymbol{v} \cdot \boldsymbol{v}\right] \mathrm{d} \Omega-\int_{\Omega} \boldsymbol{w} \cdot \nabla \cdot\left(-p \boldsymbol{I}+\mu \nabla \boldsymbol{v}+\mu(\nabla \boldsymbol{v})^{\mathrm{T}}\right) \mathrm{d} \Omega=0$.

Integration by parts (Green's Theorem) of the stress terms leads to:

$$
\begin{aligned}
& \int_{\Omega} \boldsymbol{w} \cdot \rho\left[\frac{\partial \boldsymbol{v}}{\partial t}+\nabla \boldsymbol{v} \cdot \boldsymbol{v}\right] \mathrm{d} \Omega-\underbrace{\int_{\Omega} p \nabla \cdot \boldsymbol{w} \mathrm{d} \Omega}_{\text {pressure grad. term }}+\underbrace{\int_{\Omega} \nabla \boldsymbol{w}: \mu \nabla \boldsymbol{v} \mathrm{d} \Omega}_{\text {Laplace term }} \\
& +\underbrace{\int_{\Omega} \nabla \boldsymbol{w}: \mu(\nabla \boldsymbol{v})^{\mathrm{T}} \mathrm{d} \Omega}_{\text {vel. grad. symmetrization term }}-\underbrace{\int_{\Gamma} \boldsymbol{w} \cdot\left(-p \boldsymbol{I}+\mu \nabla \boldsymbol{v}+\mu(\nabla \boldsymbol{v})^{\mathrm{T}}\right) \cdot \boldsymbol{n} \mathrm{d} \Gamma}_{\text {Divergence natural boundary term }}=0 .
\end{aligned}
$$

As in the case of the Laplace formulation, the boundary term in Eq (20) is zero because, for $\Gamma=\Gamma_{\text {no-slip }}, w$ vanishes everywhere on the boundary. Using this property and the variational form of the continuity Eq. (7), one gets the general expression defining Divergence formulations:
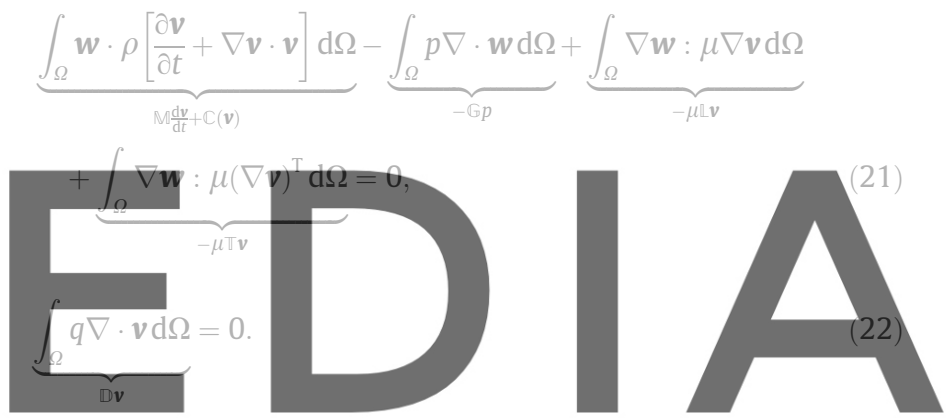

Using the same symbolic operators defined in Eqs. (16) and (17) Gownleated bew the underbraces in Eqs. (21) and (22), one

$\mathbb{M}\left(\frac{\mathrm{d} v}{\mathrm{~d} t}\right)+\mathbb{C}(\boldsymbol{v})+\mathbb{G}(p)-\mu \mathbb{L}(\boldsymbol{v})-\mu \pi(\boldsymbol{v})=0$

$\mathbb{D}(\boldsymbol{v})=0$.

Note however that a new operator $\mathbb{T}$ :

$\mu \pi(\boldsymbol{v})=-\int_{\Omega} \nabla \boldsymbol{w}: \mu(\nabla \boldsymbol{v})^{\mathrm{T}} \mathrm{d} \Omega$

coming from the symmetrization term $(\nabla \boldsymbol{v})^{\mathrm{T}}$ had to be defined.

\section{Laplace and Divergence formulations on more general boundary conditions}

Until now, we have presented two weak formulations of the NS equations the so called Laplace and Divergence formulations, both were restricted to the particular case of no-slip boundary condition $\left(\Gamma=\Gamma_{\text {no-slip }}\right)$. In that context, they are completely equivalent (except by the assumed condition of local incompressibility of the Laplace format). In this section, extensions to consider boundaries of any type: no-slip, slip and free-surfaces or any combination of them are discussed. Related issues about the boundary conditions associated with the Divergence and Laplace forms were addressed in $[40,41]$.

In order to explain the underlying concepts as simple as possible, we introduce the following mathematical property:

Weak form property: Due to the admissibility properties of $\boldsymbol{w}$ (components of $\boldsymbol{w}$ vanish where corresponding components of $\boldsymbol{v}$ are 
imposed) and the homogenous physical boundary conditions given in Eqs. (11) and (12), the following boundary term always vanishes:

$$
\int_{\Gamma} \boldsymbol{w} \cdot \overbrace{\left(-p \boldsymbol{I}+\mu \nabla \boldsymbol{v}+\mu(\nabla \boldsymbol{v})^{\mathrm{T}}\right)}^{\sigma} \cdot \boldsymbol{n} \mathrm{d} \Gamma=0
$$

for

$$
\left\{\begin{array}{c}
\text { no-slip } \\
\text { slip } \\
\text { free-surface }
\end{array}\right\} \text { boundaries }
$$

or for any boundary formed by any combination of them.

The weak form property just introduced permits us to affirm that the Divergence formulation, shown in symbolic form in Eq. (23) and repeated here:

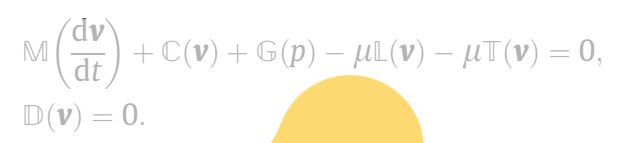

is valid not only for the case of no-slip boundaries, but also for any type of physical boundary condition. This affirmation can be proved in a straightforward way using that, when the general boundary conditions are imposed through Eq. (25), the contribution of the boundary term of Eq. (20) is still null. Thus, neither a special mod-

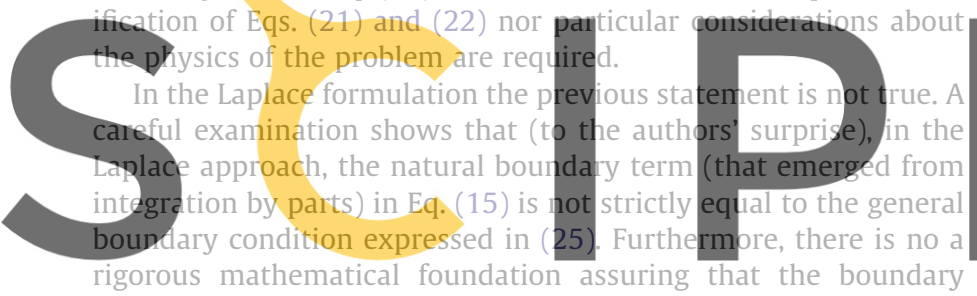
term of Eq. (15) vanishes identically for the other (i.e. slip and

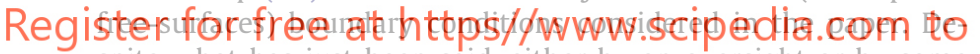
spite what has just been said, either by an oversight or by some type of empirical assumption, it is still a standard practice to not consider such natural surface contribution. In viords, this means that the Laplace formulation, shown in symbolic form in Eq. (18), and repeated here:

$\mathbb{M}\left(\frac{\mathrm{d} \boldsymbol{v}}{\mathrm{d} t}\right)+\mathbb{C}(\boldsymbol{v})+\mathbb{G}(p)-\mu \llbracket(\boldsymbol{v})=0$,

$\mathbb{D}(\boldsymbol{v})=0$.

is commonly used in general boundary conditions.

Furthermore, such unchanged form has gained popularity in the computational scientific community for general applications and also has been used as the underlying formulation for subsequent developments (projection methods, fractional-steps algorithms). With respect to this last point, we should mention that, although a number of stabilized fractional-step formulations for different velocity-pressure interpolations have been proposed based on Divergence formulations (see, for example, $[38,39,42]$ ), Laplace formulations are what we see most of the time in fractional-step algorithms. Similarly, Laplace formulations are what we see most often in pressure-projection methods.

As a conclusion of the Section we say that both formulations, the Divergence and the Laplace ones, have been widely adopted. It is not rare to see research articles where both forms are presented and discussed in parallel (see for example [14]). Besides, from the point of view of the numerical implementation, most commercial codes and open-source codes [7,11,28,29,37] are based on one or the other.

\section{Numerical tests: the revealing of non-physical solutions generated by Laplace formulations}

Recently in [20] and [21], a theoretical evidence demonstrating that Laplace formulations have a potentially severe inconsistency, has emerged. Based on that evidence, in this section a series of carefully designed simple flow tests are presented. The idea behind these tests is to see if we can capture, in real numerical experiments, the failure of the Laplace approach. For this purpose, such formulation is compared in parallel with the Divergence one. It is shown that in some of these experiments, the Laplace scheme coincides with the Divergence approach giving both correct physical answers. However in other tests, named objectivity tests, nonphysical solutions are indeed detected. We will delay to Section 7 any theoretical discussion, where we will also explain how the numerical failures can be attributable to the non-objectivity of the formulation.

The first two tests presented in this section are based on the classical square cavity problem. The two following ones are based on the study of annular cavity flows. Both of these tests have proved to be extremely useful for the purpose of this work. After these examples, other three valuable numerical tests will be shown. The simulations that are presented here have been obtained using the open-source PETSc-FEM library [29]. Its monolithic Navier-Stokes module was modified to solve the FEM equations corresponding either to Laplace formulations or to Divergence formulations. In all the examples, mixed $(\boldsymbol{v}, p)$ linear

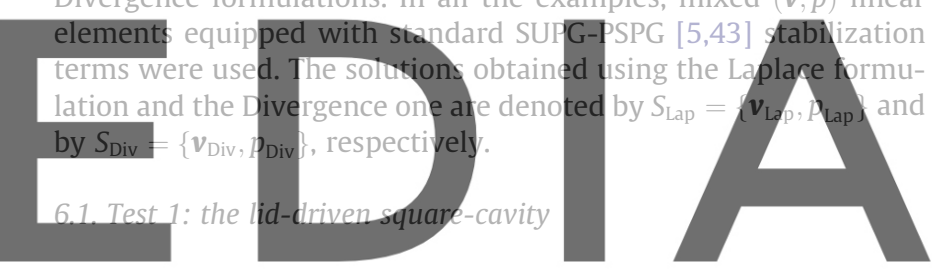

The lid-driven square-cavity is a classical test of computational fluid dynamics to assess the performance of numerical codes (see

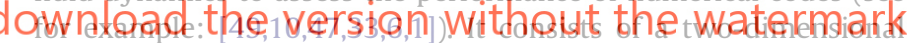
square domain filled with an incompressible fluid. The left, right and bottom boundaries are formed by no-slip fixed walls, while the upper boundary is formed by a no-slip moving wall which has an imposed horizontal velocity $U=1$. The length of the walls is chosen to be $L=1$. The two upper corners are maintained fixed. The case is shown in Fig. 1.

The purpose is to check if the same steady solution is obtained using either the Laplace or the Divergence approach. The mesh is formed by $100 \times 100$ quadrangular elements. The Reynolds number is set to $R e=1$, so as to test the case where viscous terms and convective (inertial) terms are equally important. The pressure is set to zero at the bottom right corner. The numerical results are shown in Figs. 1 and 2. The resulting streamlines using the Laplace formulation are displayed in Fig. 1 (a similar plot is recovered if the

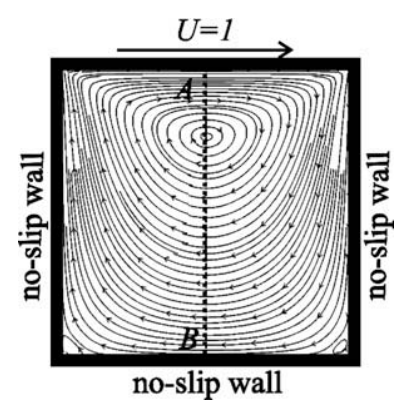

Fig. 1. The lid-driven square-cavity problem. 

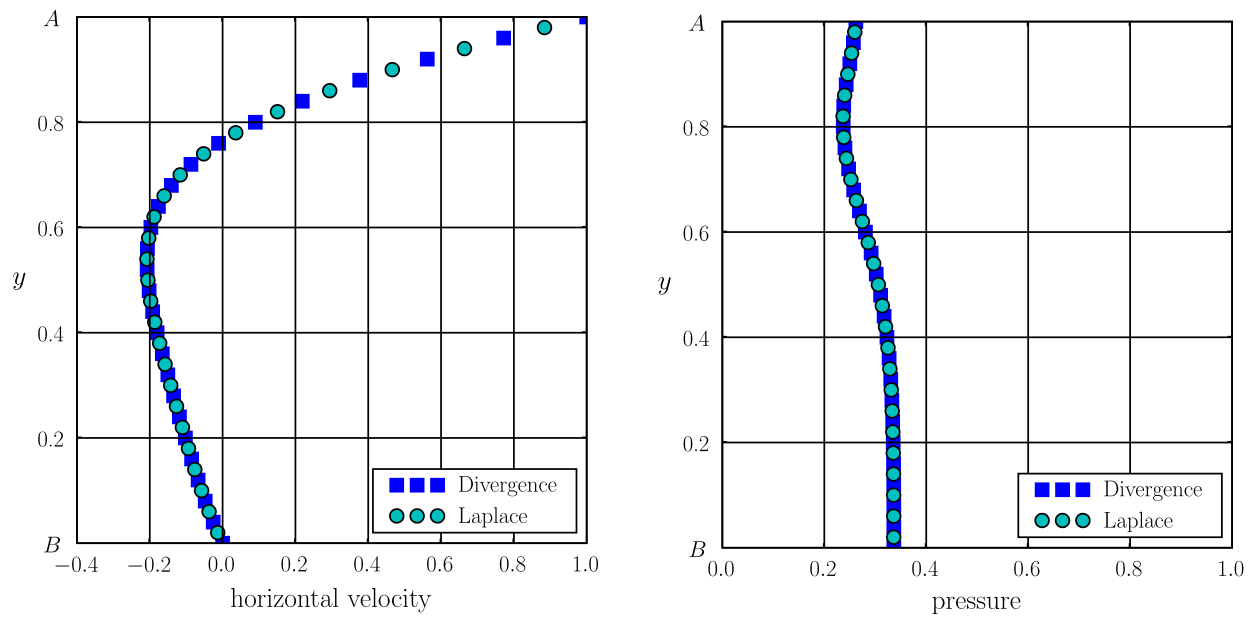

Fig. 2. The lid-driven square-cavity problem: horizontal velocity and pressure profiles (u,p) along line AB of Fig. 1

Divergence formulation is used). The left plot of Fig. 2 shows the horizontal velocity $u$ profile along the line $A B$ of Fig. 1 . From the plot it can be seen that the same velocity profile is obtained in both approaches. Observe that the horizontal velocity becomes $u=U=1$ at the upper wall and zero at the lower wall. The vortex center is located at about $y \approx 0.75$. This position approximately corresponds to the intersection of the velocity profile with the ver-

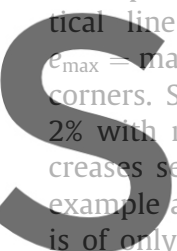
$=0$. The maximum a $\left(\left\|v_{\text {Div }}-v_{\text {Lap }}\right\|\right)$, is about 0.02 Such value corresponds to a respect to the reference veloc everal orders of magnitude along the line $A B$, the velocity's $0.004 \%$. Note from the plot on the right of Fig. 2 that the pressure values also match well; along the line AB the pressure's maximum absolute error is 0.001 .

\section{Register for free at https//www.scipedia.com to d 6.2. Test 2: the slippery square-cavity}

A good idea to check both formulations under silip boundary conditions is to modify the standard lid-driven square-cavity, defined in test 1 , by changing the no-slip condition to a slip condition in the three non-driven walls (i.e. the left, right and bottom walls). In Figs. 3 and 4, the obtained numerical results are shown. The streamlines corresponding to the solution obtained using the Divergence formulation are displayed in Fig. 3 (a similar plot can be recovered for the Laplace one).

Fig. 4 shows plots of the horizontal velocity $u$ and the pressure, along the line $\mathrm{AB}$ of Fig. 3 . It can be seen that the same velocity and pressure profiles are obtained for both formulations. Note from the left plot that, because of the slip condition, the velocities on the non-driven walls are non-zero now (see velocity $u$ at point $B$ ).

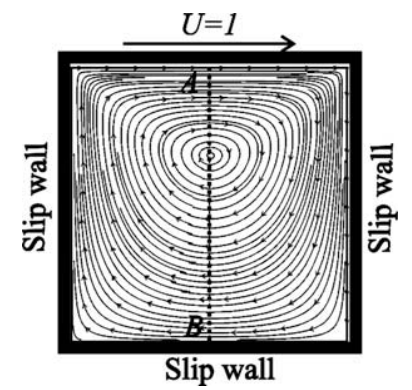

Fig. 3. The slippery square-cavity problem
The vortex center moves down to about $y \approx 0.68$, corresponding to the intersection of the velocity profile with the vertical line $u=0$. Again, the maximum relative error in velocities, $e_{\max }=\max \left(\left\|\boldsymbol{v}_{\text {Div }}-\boldsymbol{v}_{\text {Lap }}\right\|\right)$, in all the domain is less than $2 \%$ with respect to the reference velocity $U$ and occurs near the upper corners. As in the test 1 , these differences can be attributed to the flow singularity occurring near these two areas. In the other regions of the

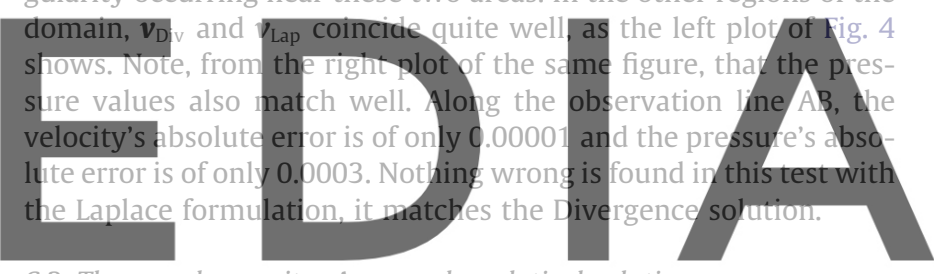

6.3. The annular-cavity: A general analytical solution

dowseload stherversionbwithoutt thenewaterstmark tempts to detect the non-objectivity of Laplace formulations failed: running the well-known and commonly used square-cavity test does not show any reaily appreciable discrepancy between Laplace and Divergence solutions. Is the error in Laplace formulations so small and negligible to just really be appreciable in extreme cases with free-surfaces as the ones shown in [21]? Are there no simpler tests that could clearly show the effects of the violation of objectivity and that be simple enough so that any researcher working in numerical simulations of fluids could replicate? This topic is addressed in what follows.

Flows between cylinders are commonly used for studies of viscometry, hydrodynamic stability, lubrication and bifurcation theory, etc. (see for example: [4,35,24,25]). Among these different types of flows, here, the case of flows in the annular cavity is considered. The annular-cavity problem consists of the study of 2D flows (fluid motion in the axial direction is assumed zero) between two coaxial cylindrical walls, as shown in Fig. 5. The internal cylinder has a radius $r_{\mathrm{A}}$ and the external cylinder a radius $r_{\mathrm{B}}$, both can be arbitrary. These series of tests have two fundamental properties. First, one can easily set up different boundary conditions to the walls, such as imposing different angular velocities together with slip or no-slip conditions. Second, analytical solutions can be derived for all these different types of boundary conditions.

Let us obtain the general analytical solution of the NavierStokes Equations for the case of two-dimensional steady laminar flows around two coaxial cylinders of arbitrary radius, $r_{\mathrm{A}}$ and $r_{\mathrm{B}}$, respectively. In polar coordinates $(r, \theta)$ the $2 \mathrm{D}$ steady NavierStokes Eqs. (1) and (2) can be written as: 

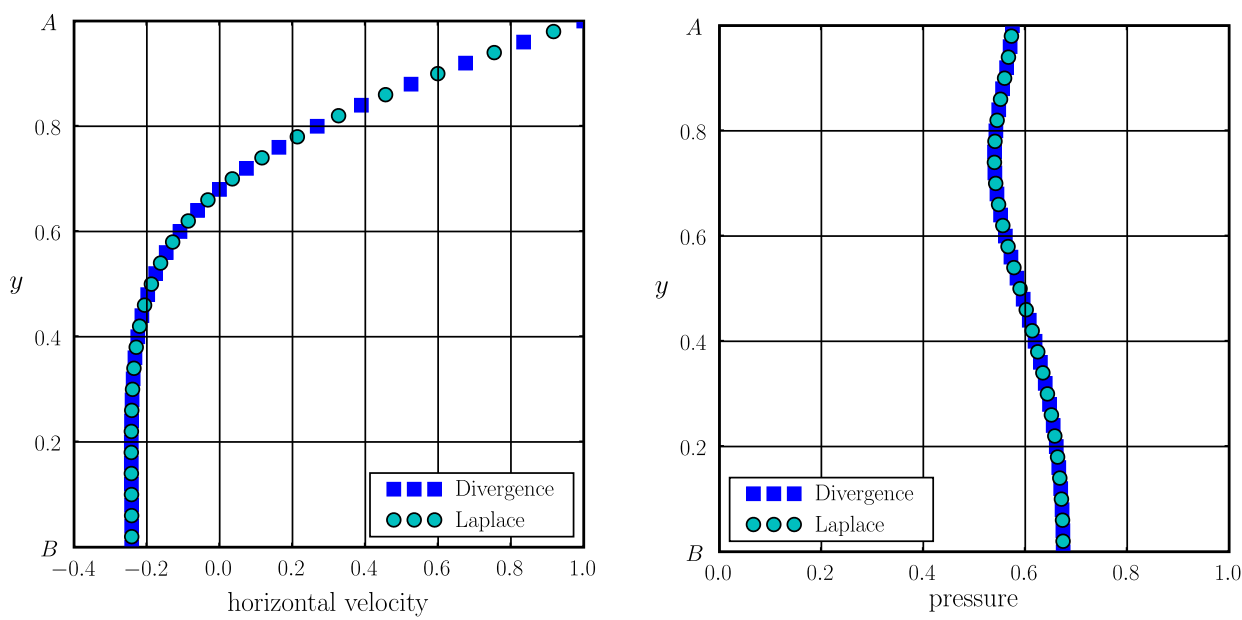

Fig. 4. The slippery square-cavity problem: horizontal velocity and pressure profiles $(u, p)$ along line AB of Fig. 3.
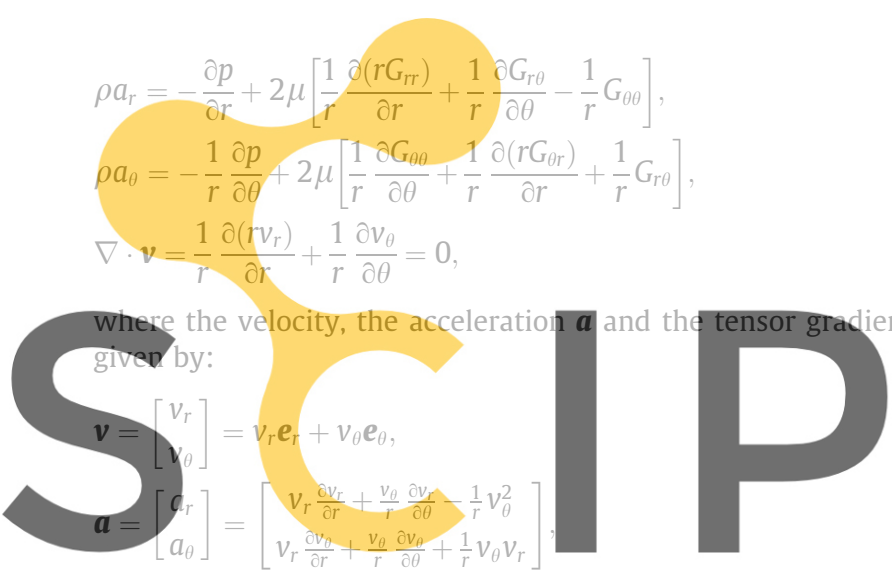

(28)

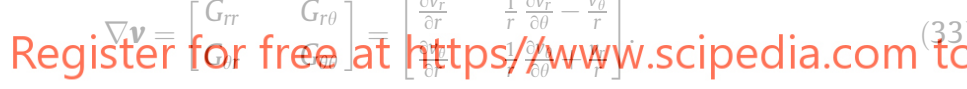

By symmetry, it can be assumed that the flow fields do not depend on 0 , i.e.:

$v_{r}=v_{r}(r) ; \quad v_{\theta}=v_{\theta}(r) ; \quad p=p(r)$.

Inserting Eq. (34) into the continuity Eq. (30) and using the condition of non-permeable cylinder walls, we get that:

$v_{r}=0$ in all the domain.

Taking into account (34) and (35), the tensor gradient reduces to:

$\nabla \boldsymbol{v}=\left[\begin{array}{ll}G_{r r} & G_{r \theta} \\ G_{\theta r} & G_{\theta \theta}\end{array}\right]=\left[\begin{array}{cc}0 & -\frac{v_{\theta}}{r} \\ \frac{\partial v_{\theta}}{\partial r} & 0\end{array}\right]$

and the momentum equations simplify to:

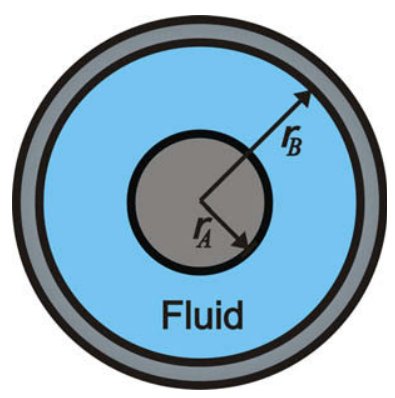

Fig. 5. The annular-cavity problem.

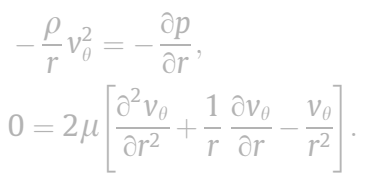

Solving Eq. (38) for $v_{\theta}$ and Eq. (37) for $p$, the general solution for the flow is obtained:

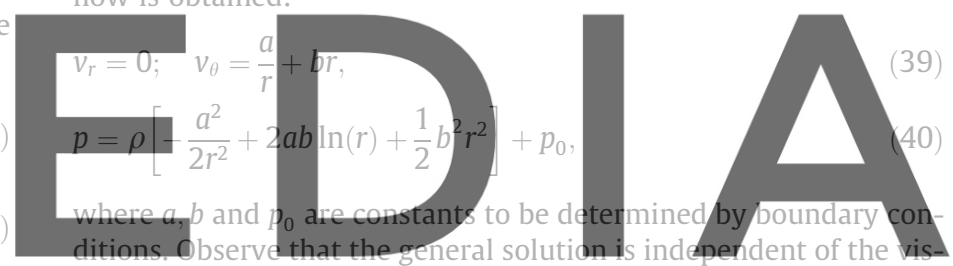

cosity $\mu$, i.e. all steady flow solutions are independent of the

\section{download the version without the watermark}

6.4. Test 3: the fully-driven annular-cavity

Now let us obtain the particular solutions corresponding to fully-driven flows in the annular cavity. These solutions correspond to the case when both cylinders are rotating with arbitrary angular velocities $\omega_{\mathrm{A}}$ and $\omega_{\mathrm{B}}$, and their walls are no-slip walls, as shown in Fig. 6 . Then, the following boundary conditions apply:

$v_{\theta}\left(r_{\mathrm{A}}\right)=V_{\mathrm{A}}=\omega_{\mathrm{A}} r_{\mathrm{A}} ; \quad v_{\theta}\left(r_{\mathrm{B}}\right)=V_{\mathrm{B}}=\omega_{\mathrm{B}} r_{\mathrm{B}} ; \quad p\left(r_{\mathrm{A}}\right)=0$.

Remark 2. The fully-driven annular-cavity problem presented in this section can be seen as the 2D case of the renowned TaylorCouette flow $[36,26]$.

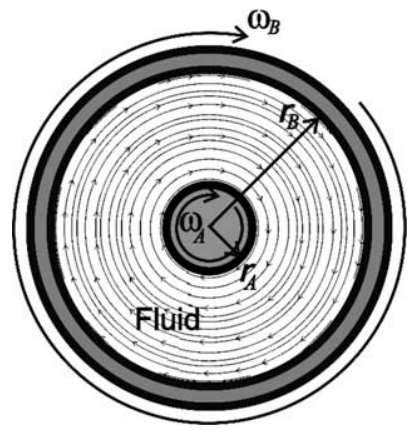

Fig. 6. The fully-driven annular-cavity problem. 

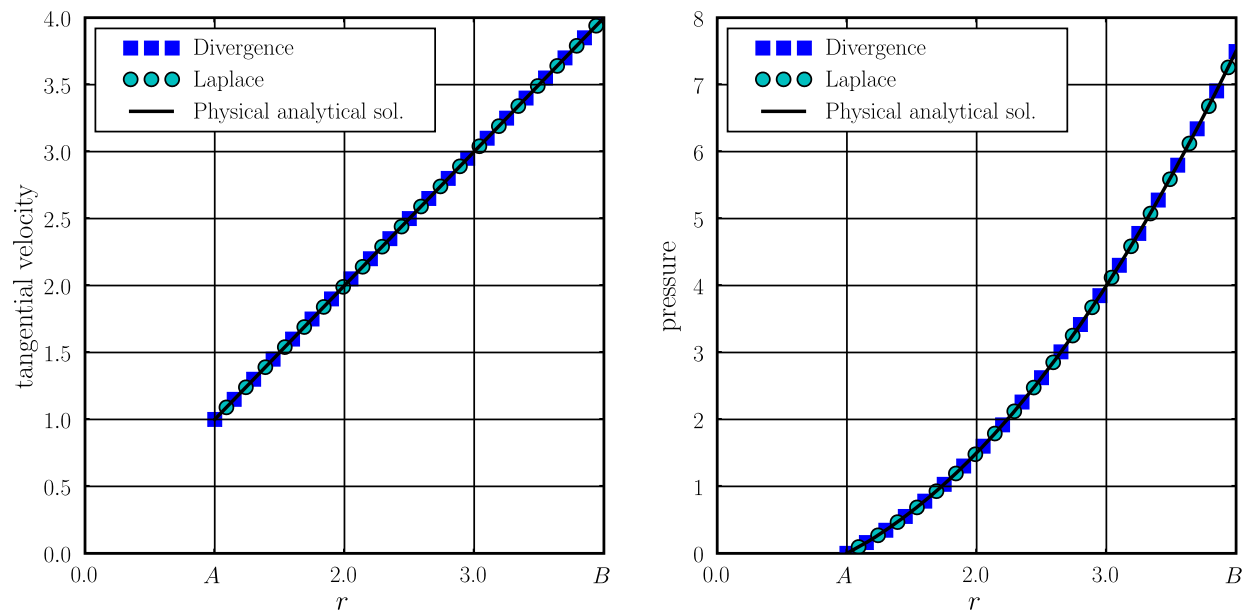

Fig. 7. The fully-driven annular-cavity problem: tangential velocity and pressure profiles $\left(v_{0}, p\right)$ along the radial coordinate $r$.

Inserting Eq. (41) into Eqs. (39) and (40), it can be shown that the general solution for the fully-driven cavity is given by:

$a=-\frac{r_{\mathrm{B}}^{2} r_{\mathrm{A}}^{2}}{\left(r_{\mathrm{B}}^{2}-r_{\mathrm{A}}^{2}\right)}\left(\omega_{\mathrm{B}}-\omega_{\mathrm{A}}\right) ; \quad b=\frac{\omega_{\mathrm{B}} r_{\mathrm{B}}^{2}-\omega_{\mathrm{A}} r_{\mathrm{A}}^{2}}{\left(r_{\mathrm{B}}^{2}-r_{\mathrm{A}}^{2}\right)}$

When both cylinders rotate with the same angular velocity

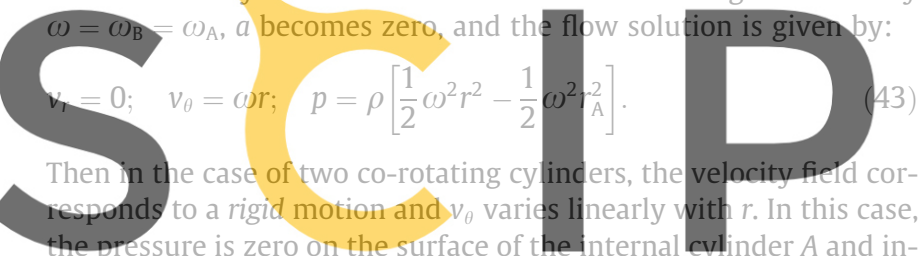

creases quadratically with $r$. The increase in pressure is an effect of

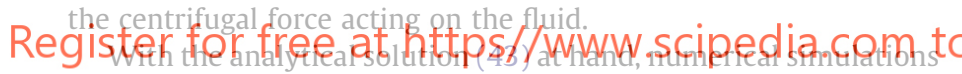

were done to see if differences between Divergence and Laplace formulations can be detected. Figs. 6 and 7 show the numerical results obtained for the case where $r_{\mathrm{A}}=1, r_{\mathrm{B}}=4$ and $\omega=\omega_{\mathrm{B}}=$ $\omega_{\mathrm{A}}=1$. In Fig. 6 , the obtained streamlines for the Divergence case are displayed. A similar plot can be recovered for the Laplace case. From Fig. 7, one can see that both solutions behave equally well matching the analytical solution.

\subsection{Objectivity test 1: the slippery annular-cavity}

In this section, the results corresponding to slippery annularcavity flow problems are presented. These particular flows are obtained when the internal cylinder is formed by a no-slip wall that is rotating with an arbitrary angular velocity $\omega_{\mathrm{A}}$, while the external

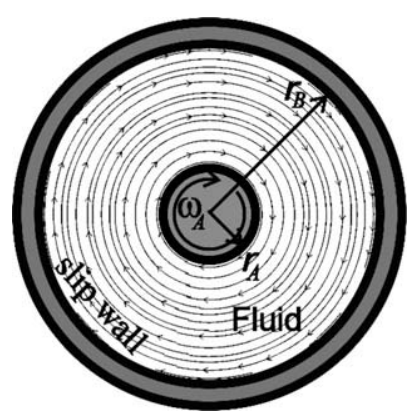

Fig. 8. The slippery annular-cavity problem. cylinder is formed by a slip wall. The case is shown in Fig. 8 and corresponds to the following boundary conditions:

$v_{\theta}\left(r_{\mathrm{A}}\right)=V_{\mathrm{A}}=\omega_{\mathrm{A}} r_{\mathrm{A}} ; \quad\left[\boldsymbol{e}_{\theta} \cdot\left(\boldsymbol{\sigma} \cdot \boldsymbol{e}_{r}\right)\right]_{r=r_{\mathrm{B}}}=0 ; \quad p\left(r_{\mathrm{A}}\right)=0$

where the second equation corresponds to the slip condition on the external cylinder $B$. The analytical solution for this type of flows can

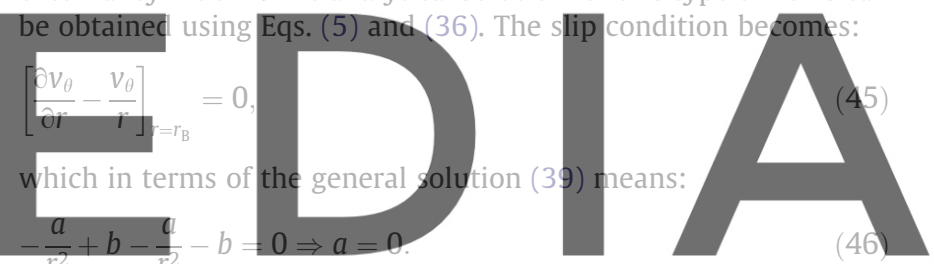

Then, the closed-form expression for the case of slippery annulardow whead: the version without the watermark

$v_{r}=0 ; \quad v_{\theta}=\omega_{\mathrm{A}} r ; \quad p=\rho\left[\frac{1}{2} \omega_{\mathrm{A}}^{2} r^{2}-\frac{1}{2} \omega_{\mathrm{A}}^{2} r_{\mathrm{A}}^{2}\right]$

Comparison of Eqs. (43) and (47) tell us that the slippery annularcavity and the fully-driven annular-cavity with co-rotating cylinders $\left(\omega=\omega_{\mathrm{B}}=\omega_{\mathrm{A}}\right)$ gives the same steady flow solution: a rigid motion of the fluid. Observe again that this behavior is independent of the Reynolds number.

Next, the numerical results obtained for the case $r_{\mathrm{A}}=1, r_{\mathrm{B}}=4$ and $\omega_{\mathrm{A}}=1$ are shown in Fig. 9. According to Eq. (47) a linearly increasing tangential velocity and a quadratic variation of pressure should be obtained. In particular for the velocity we expect to get the value $v_{\theta}\left(r_{\mathrm{B}}\right)=4.0$ on the external cylinder. From the results shown in Fig. 9 one can clearly see that, while the Divergence scheme still matches the analytical solution, the Laplace formulation gives a completely wrong answer. Note the loss of physical meaning of the Laplace solution: the tangential velocity starts at the imposed velocity on the inner cylinder $V_{\mathrm{A}}=1$, but instead of increasing linearly with the distance from the origin $r$, it continually decreases to a value $v_{\theta}\left(r_{\mathrm{B}}\right) \approx 0.47$. A similar phenomenon can be observed for the pressure: the expected quadratic increase due to the centrifugal force is not recovered. This simple objectivity test shows the severe consequences of the violation of objectivity. Some more tests are presented next.

\subsection{Objectivity test 2: the slippery semicircular-cavity}

The lid-driven semicircular-cavity is another test of computational fluid dynamics used as benchmark of numerical schemes 

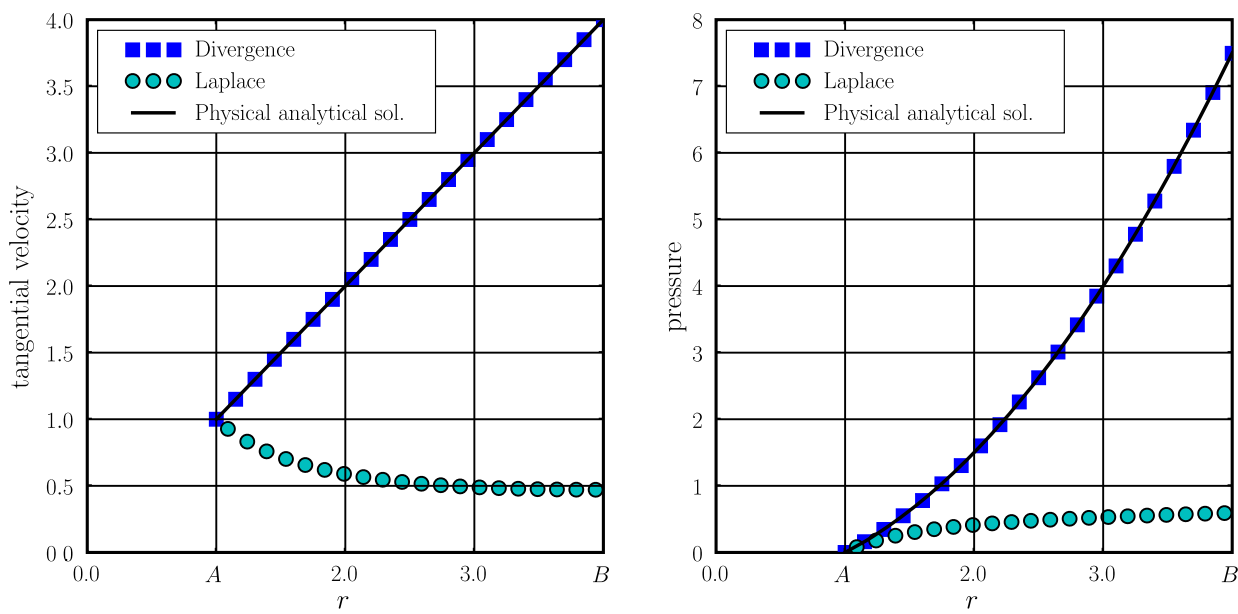

Fig. 9. The slippery annular-cavity problem: tangential velocity and pressure profiles $\left(v_{\bullet}, p\right)$ along the radial coordinate $r$.

and in the context of studies of bifurcation theory [12,18]. It consists of a 2D semicircular domain filled with an incompressible fluid. In the standard test the semicircular boundary is formed by a fixed no-slip wall, while the upper rectilinear boundary, i.e the lid, is formed by a moving wall which has an imposed horizontal velocity $U=1$. The radius of the semicircular wall is chosen to be $r_{\mathrm{B}}=1$. The two upper corners are maintained fixed. When this

\section{classical test is numerically solved, $S_{I}$ an and $S_{\text {Div }}$ agree in the same}

Wayas the previously discussed fully-driven

and annular-cavity).

For the purpose of detecting the failure of Laplace formulations, the standard test is modified by changing the no-slip condition, on the semicircular wall, to a slip condition, as depicted in Fig. 10. The

Register for free at https

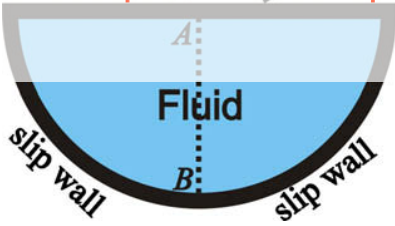

Fig. 10. The slippery semicircular-cavity problem.

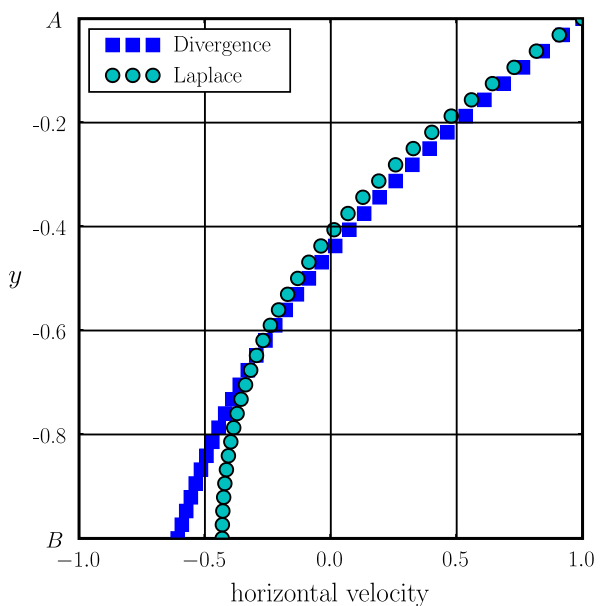

numerical values of the horizontal velocity $u$ along the line $A B$ are shown in the left plot of Fig. 11. Observe that, although both solutions match the prescribed condition $u_{\text {Lap }}=u_{\text {Div }}=1$ on the upper boundary, the velocity profile along the line is different. One remarkable property of the Laplace scheme is that the gradient of the velocity field tends to zero near the slip wall. Note that different pressure profiles are obtained too (see right plot of Fig. 11).
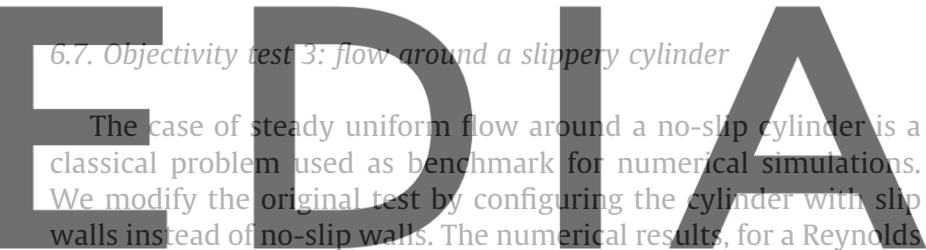

number $R e=1$ and an incident horizontal velocity $V=1$, are shown in Figs. 12 and 13. In Fig. 12, the resulting streamlines for

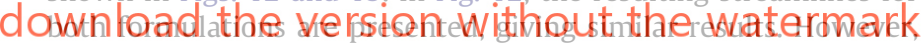
when the velocity $V$ and pressure profiles along the upper semicircular arc ABC are plotted, severe discrepancies are observed (see Fig. 13). The most notable difference is that the flow accelerates more in the Laplace solution, reaching a maximum speed of about 1 instead of the value close to 0.6 obtained when the Divergence formulation is employed. Note that for this case the effect of non-objectivity is similar as if an external force would be helping the flow to move along the cylinder.

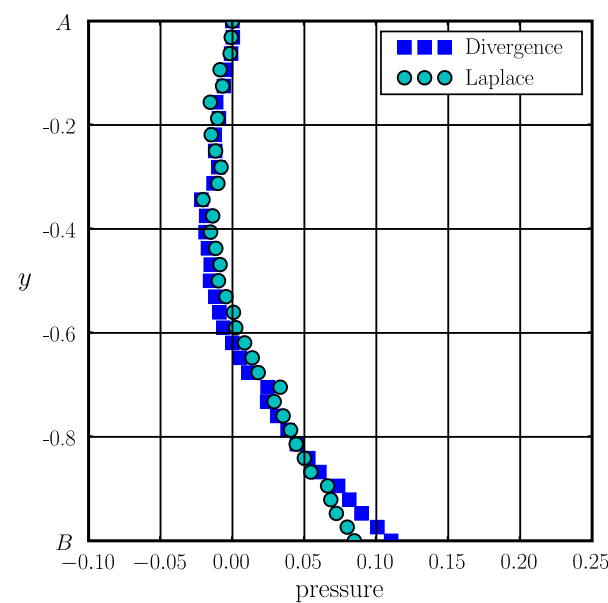

Fig. 11. The slippery semicircular-cavity problem: horizontal velocity and pressure profiles (u,p) along line AB of Fig. 10 . 


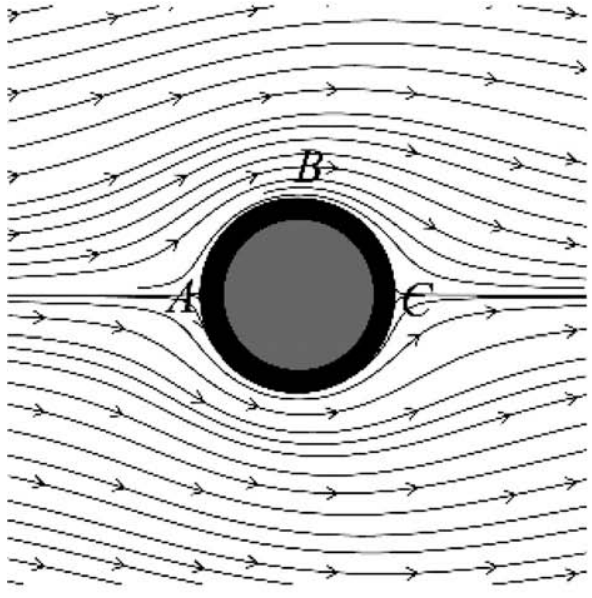

(a) Diveruence Form

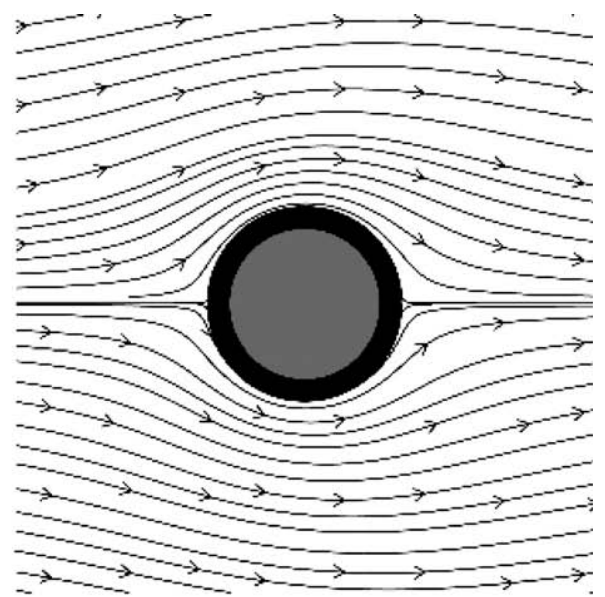

(b) Laplacian Form

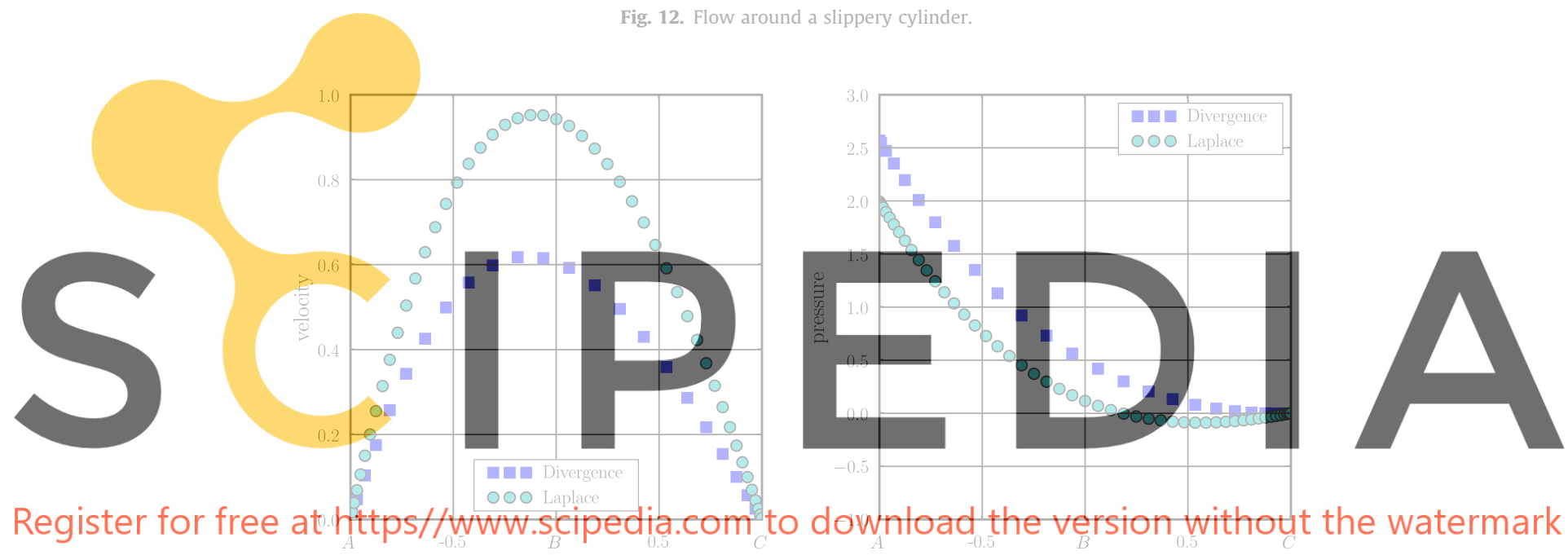

Fig. 13. Flow around a slippery cylinder: velocity profiles and pressure profiles $(\|v\|, p)$ along the semicircular arc ABC shown in Fig. 12.

\subsection{Objectivity test 4: a free-surface sloshing test}

The last test presented is the sloshing problem, i.e., a problem with free-surfaces, depicted in Fig. 14. It consists of a tank filled with liquid at rest such that, at time $t=0$, its free-surface is configured so as to have a sinusoidal shape perturbation. As a consequence of the perturbation, the free-surface of the liquid moves in an oscillatory motion [2,32]. For small amplitudes an analytical solution can be obtained [31].

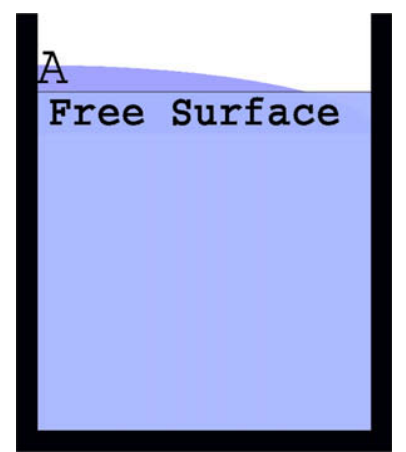

Fig. 14. Sloshing tank problem.
Following the same configuration set-up defined in [2], the problem is run using both formulations. The obtained results, for the motion of the fluid particle $A$ on the free-surface, are displayed in Fig. 15. Note that the Divergence scheme agrees quite well with

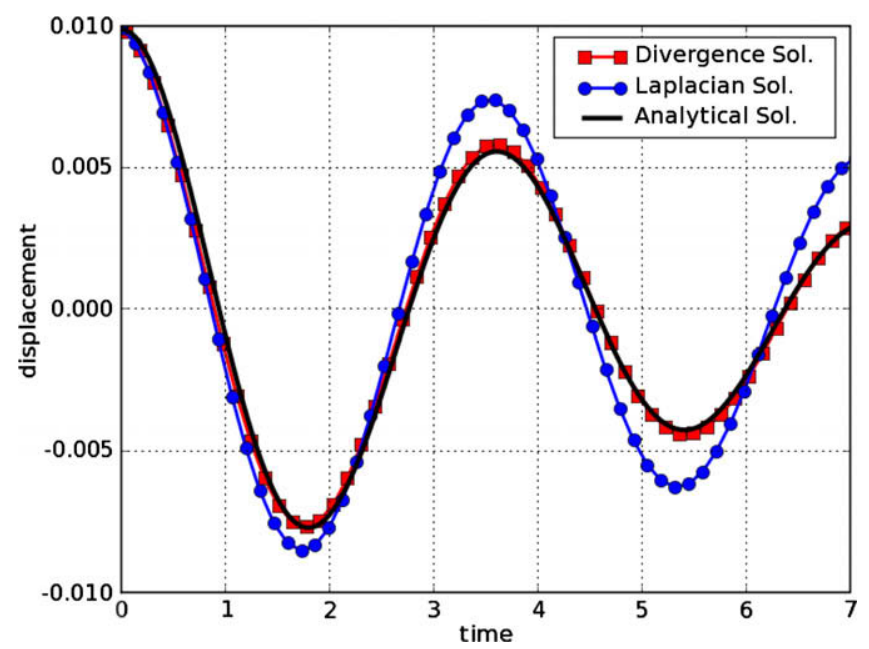

Fig. 15. Sloshing tank problem: time evolution of the fluid particle located at point A on the free surface, as depicted in Fig. 14 
the analytical solution while the Laplace approach notably differs from it, having a slightly higher frequency and a much smaller damping.

\section{Discussion of results}

In the previous section a number of tests were presented. Among them, the named objectivity tests reveal a series of nonphysical solutions obtained when Laplace formulations are used.

\subsection{A correct approach: the corrected Laplace formulation}

After seen the numerical solutions obtained by Laplace formulations, we do not want the reader to fall into the conclusion that starting from the Laplace format (1) and (2) of the NS equations is definitely wrong. Although we recommend the Divergence approach because is more general, a correct formulation can indeed be obtained from the Laplace approach. This is shown in this section. We have named the correct approach: corrected Laplace formulation, to make it clear that it is the counterpart of the Laplace formulation and to emphasize that it is not the standard or classical approach.

The correct formulation valid for the three types of boundary conditions (no-slip, slip or free-surfaces or for any boundary that is a combination of them) can be obtained by taking into account the natural boundary integral of Eq. (15) (which was neglected in the derivation of the Laplace formulation in Section 5). Such

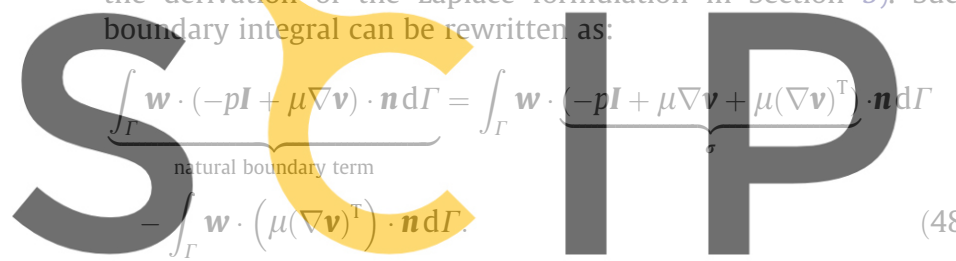

Then, after imposing the physical boundary conditions through the

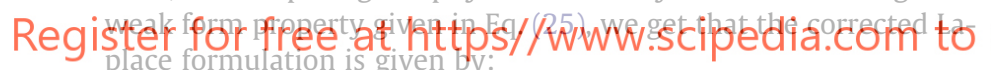

$$
\begin{aligned}
& \int_{\Omega} w \cdot \rho\left[\frac{\partial v}{\partial t}+\nabla v \cdot v\right] \mathrm{d} \Omega-\underbrace{\int_{\Omega} p \nabla \cdot w \mathrm{~d} \Omega}_{\text {pressure grad. term }}+\underbrace{\int_{\Omega} \nabla w: \mu \nabla v \mathrm{~d} \Omega}_{\text {Laplace term }} \\
& +\underbrace{\int_{\Gamma} \boldsymbol{w} \cdot\left(\mu(\nabla \boldsymbol{v})^{\mathrm{T}}\right) \cdot \boldsymbol{n} \mathrm{d} \Gamma}_{\text {additional boundary term }}=0 .
\end{aligned}
$$

If we define the symbolic operator $\mathbb{V}$ :

$\mu \mathbb{V}(\boldsymbol{v})=-\int_{\Gamma} \boldsymbol{w} \cdot\left(\mu(\nabla \boldsymbol{v})^{\mathrm{T}}\right) \cdot \boldsymbol{n} \mathrm{d} \Gamma=0$

and use the operators defined in Section 3, we can write the corrected Laplace formulation in symbolic form as:

$\mathbb{M}\left(\frac{\mathrm{d} \boldsymbol{v}}{\mathrm{d} t}\right)+\mathbb{C}(\boldsymbol{v})+\mathbb{G}(p)-\mu \llbracket(\boldsymbol{v})-\mu \mathbb{V}(\boldsymbol{v})=0$,

$\mathbb{D}(\boldsymbol{v})=0$.

Note that the corrected Laplace formulation does not longer have the property that the viscous terms are given as function of the Laplace diffusion operator only.

To see the numerical behavior of the corrected formulation, we implemented the scheme into PETSc-FEM (by adding the additional boundary term to the existent Laplace module) and re-run the tests of Section 6. In Figs. 16 and 17, we show as an example, the results corresponding to the cases of the slippery annular-cavity and of the flow around a slippery cylinder (Sections 6.5 and 6.7, respectively). As can be seen from these figures, now, the corrected Laplace scheme gives right physical responses. Note that such solutions also match the ones of the Divergence approach.

The agreement between the corrected Laplace and the Divergence formulations can, indeed, be proved mathematically in the continuum from the following operator equality (derived using integration by parts and the incompressibility equation):
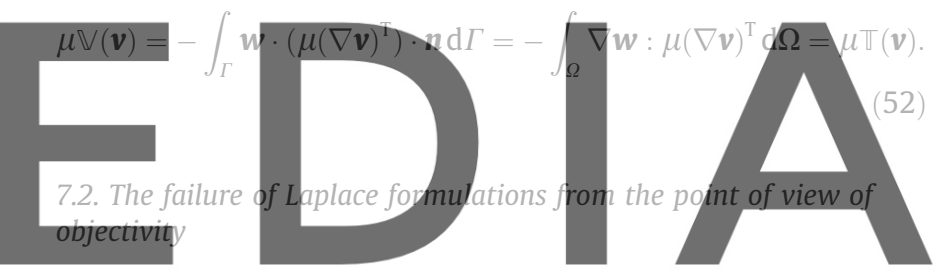

Comparison of the Laplace formulation given in Eq. (27) with

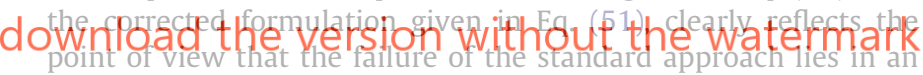
inappropriate management of boundary conditions. Not imposing the real physical conditions caused the absence of a integral boundary term. As shown in the numerical tests, the missing of such term produces severely distorted non-physical solutions.

However, we want to emphasize that the failure of the Laplace formulation was not detected by realizing that there was a problem with the imposed boundary conditions. After all, the use of
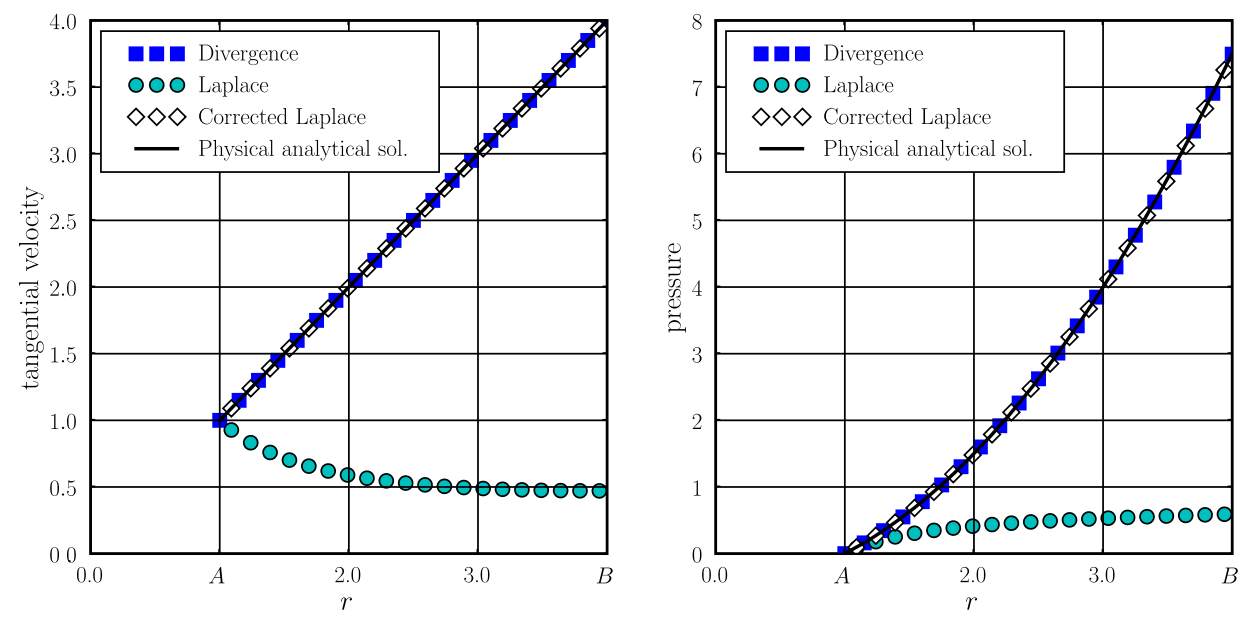

Fig. 16. The slippery annular-cavity problem. Solutions obtained with the three formulations: Divergence, Laplace and corrected Laplace. 

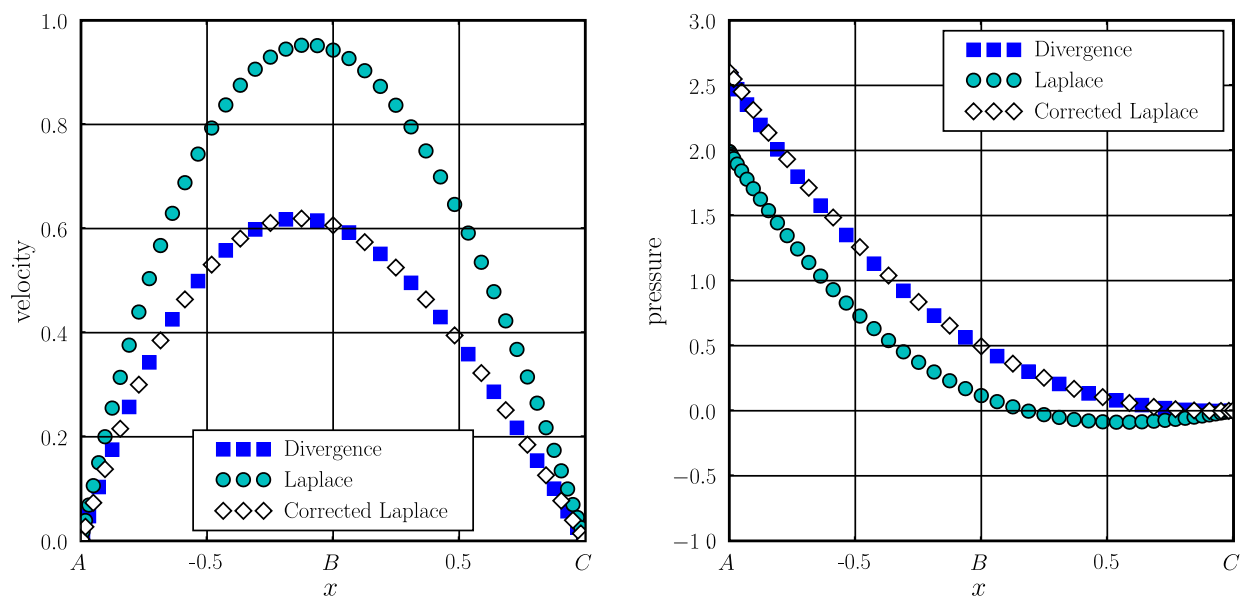

Fig. 17. Flow around a slippery cylinder. Solutions obtained with the three formulations: Divergence, Laplace and corrected Laplace.

approximate boundary conditions is very common in physics and computational fluid mechanics. Actually, the discovery of the failure came by noticing that the Divergence formulation was able to give correct physical responses where the Laplace one did not. This provided an alternative point of view which shows that the nonphysical responses of the Laplace formulation can be explained as caused by its lack of objectivity.

As mentioned above, such objective point of view surges from the comparison of the Laplace formulation (27) with the Divergence formulation (26). Since the Divergence gives corrects physical answers, the comparison leads to the conclusion that the failure of the Laplace formulation can be seen as caused by a missing volume integral:

$\mu \rrbracket(\boldsymbol{v})=-\int_{\Omega} \nabla \boldsymbol{w}: \mu(\nabla \boldsymbol{v})^{\mathrm{T}} \mathrm{d} \Omega$.

The observation that this volume integral comes from the symmetrization of the velocity gradient in the constitutive Eq. (5), was the key factor for the demonstration that Laplace formulations are nonobjective [20,21]. In particular, it can be rigorously proved that such type of formulation does not model anymore the correct fluid material given by Eq. (5), but instead it models a non-objective material whose constitutive equation is given by:

$\tilde{\boldsymbol{\sigma}}=-p \boldsymbol{I}+\mu \nabla \boldsymbol{v}$.
If this equivalent point of view is adopted, then the non-physical solutions obtained with Laplace formulations can be seen as a natural manifestation of the non-objectivity of the simulated material defined in Eq. (54). We want to remark, again, that the numerical tests were designed to get such manifestation in the whole fluid domain and not by paying particular attention to boundary effects.

In Section 6.5, it was observed that Laplace formulations give a severely incorrect answer for the case of slippery annular-cavity tests. As pointed out in the previous paragraph, using such type of formulations is equivalent to simulate a non-objective fluid material which does not have the symmetrization of the stress tensor. It is possible, indeed, to get the analytical solution that describe the behavior of this non-physical fluid.

From the definition of the non-objective stress tensor in Eq. (54) and the equations of conservation of mass and momentum, it can be seen that such non-objective fluid still satisfies the incompressible NS Eqs. (1) and (2). As a consequence, for the slippery annularcavity, its response can be obtained by solving the NS equations but with an erroneous traction boundary condition:

$\left.v_{\theta}\left(r_{\mathrm{A}}\right)=\omega_{\mathrm{A}} r_{\mathrm{A}} ; \quad[\boldsymbol{e}_{\theta} \cdot \underbrace{(-p \boldsymbol{I}+\mu \nabla \boldsymbol{v})}_{\tilde{\sigma}} \cdot \boldsymbol{e}_{\mathrm{r}})\right]_{r=r_{\mathrm{B}}}=0 ; \quad p\left(r_{\mathrm{A}}\right)=0$.
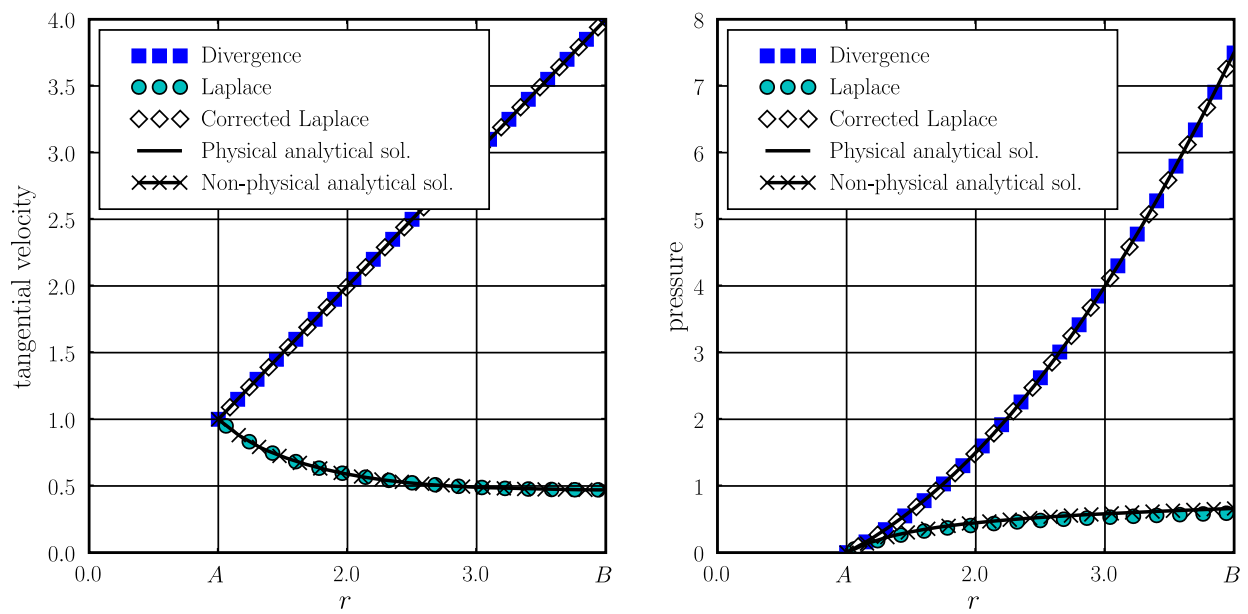

Fig. 18. The slippery annular-cavity problem. The non-physical analytical solution corresponding to the non-objective fluid material defined in Eq. (54) is included. 
Using Eq. (36), the erroneous traction condition becomes:

$\left[\frac{\partial v_{\theta}}{\partial r}\right]_{r=r_{\mathrm{B}}}=0$

Then, inserting Eqs. (55) and (56) into Eqs. (39) and (40), one can get the analytical solutions that corresponds to the non-physical fluid material defined by the Laplace approach:

$a=\frac{\omega_{\mathrm{A}} r_{\mathrm{B}}^{2} r_{\mathrm{A}}^{2}}{\left(r_{\mathrm{B}}^{2}+r_{\mathrm{A}}^{2}\right)} ; \quad b=\frac{\omega_{\mathrm{A}} r_{\mathrm{A}}^{2}}{\left(r_{\mathrm{B}}^{2}+r_{\mathrm{A}}^{2}\right)}$,

So:

$v_{r}=0 ; \quad v_{\theta}=\frac{\omega_{\mathrm{A}} r_{\mathrm{B}}^{2} r_{\mathrm{A}}^{2}}{\left(r_{\mathrm{B}}^{2}+r_{\mathrm{A}}^{2}\right)} \frac{1}{r}+\frac{\omega_{\mathrm{A}} r_{\mathrm{A}}^{2}}{\left(r_{\mathrm{B}}^{2}+r_{\mathrm{A}}^{2}\right)} r$.

In Fig. 18, Fig. 16 has been plotted again but adding the curve for the analytical solutions given by (57) and (58). From the new plot it is clear that the numerical solutions obtained by Laplace formulations match the analytical solutions of the non-objective fluid material.

The proof that the material being simulated is given by (54) is general and applies to the three types of boundary conditions (no-slip, slip and free-surfaces). Now, when the fluid is fully bounded by non-slip walls, i.e. $\Gamma=\Gamma_{\text {no-slip, }}$ such a non-objective material cannot be distinguished from the correct one. Mathematically, this can be seen by noting that, under the no-slip condition, the corrective term vanishes:

$[\mu \mathbb{V}(\boldsymbol{v})]_{\Gamma_{\text {no-slip }}}=0$.

Numerically, this phenomenon can be observed in tests 1 and 3 of Section 6 where both, the Laplace and the Divergence, formulations match. In other flow situations, with boundaries having slip walls or free-surfaces, one should expect to detect a difference. This is what happens in the objectivity tests.

However, the reader may ask why, in the slippery square-cavity, where a slip condition was used, both approaches gave the same result. The answer to this requires to put more attention to the integrand of the missing boundary term (50). Splitting the admissible test functions, into surface's normal and tangential coordinates

$\boldsymbol{w}=w_{t} \boldsymbol{t}+w_{n} \boldsymbol{n}$

and making use that the normal component of the test function is zero, because normal velocities are set to zero $(\boldsymbol{v} \cdot \boldsymbol{n}=0)$, one gets that:

$$
\begin{aligned}
{[\mu \mathbb{V}(\boldsymbol{v})]_{\Gamma_{\text {slip }}} } & =-\int_{\Gamma_{\text {slip }}} \mu \boldsymbol{w} \cdot(\nabla \boldsymbol{v})^{\mathrm{T}} \cdot \boldsymbol{n} \mathrm{d} \Gamma \\
& =-\int_{\Gamma_{\text {slip }}} \mu w_{\boldsymbol{t}} \frac{\partial v_{j}}{\partial x_{\boldsymbol{t}}} n_{j} \mathrm{~d} \Gamma .
\end{aligned}
$$

Using the chain rule in the last two terms of the integrand, we get that:

$[\mu \mathbb{V}(\boldsymbol{v})]_{\Gamma_{\text {slip }}}=-\int_{\Gamma_{\text {slip }}} \mu w_{\boldsymbol{t}}\left(\frac{\partial\left(v_{j} n_{j}\right)}{\partial x_{t}}-v_{j} \frac{\partial n_{j}}{\partial x_{\boldsymbol{t}}}\right) \mathrm{d} \Gamma$

Now, the first term inside the parenthesis is zero, because $\boldsymbol{v} \cdot \boldsymbol{n}=0$ along the surface's tangential direction:

$\frac{\partial\left(v_{j} n_{j}\right)}{\partial x_{t}}=\frac{\partial(\boldsymbol{v} \cdot \boldsymbol{n})}{\partial x_{t}}=0$.

So we get that:

$[\mu \mathbb{V}(\boldsymbol{v})]_{\Gamma_{\text {slip }}}=\int_{\Gamma_{\text {slip }}} \mu w_{\boldsymbol{t}}\left(v_{j} \frac{\partial n_{j}}{\partial x_{\boldsymbol{t}}}\right) \mathrm{d} \Gamma$

Now by definition the $\frac{\partial n_{j}}{\partial x_{t}}$ term is inversely proportional to the radius of curvature $R_{\text {slip }}$ of the slip wall, then we have: $[\text { missing term }]_{\Gamma_{\text {slip }}} \propto \frac{1}{R_{\text {slip }}}$.

Then for straight walls, $R_{\text {slip }} \rightarrow \infty$ and the missing term vanishes again. This explains why in the case of the slippery square-cavity both formulations give quite similar results.

Eqs. (59) and (64) explain also why the phenomenon of violation of objectivity has been undetected in many standard flow problems.

Finally, it is worth to mention that the results obtained for the sloshing tank problem prove that Laplace formulations cannot even be used in problems dealing with small perturbations.

\section{Conclusions}

In this article, a number of easily reproducible numerical tests has been presented. Running them with numerical schemes based on Laplace formulations reveal that such formulations produce severely distorted non-physical solutions. Stated in different words, these tests can make visible the non-objectivity of such formulations. Here, it is shown that although Laplace formulations give correct results in the case of fully-imposed (no-slip) domains, they yield non-physical behavior in problems dealing with flows around slip walls and flows with free surfaces.

The results obtained here are a useful reminder that boundary terms and boundary conditions should always be treated with special care.

Note, that there might be several numerical programs based on Laplace forms available to researchers and engineers, who might be unaware of the flaw of these formulations. Special attention should be given to schemes based on fractional-step or projection methods (see the reviews by Guermond et al. $[13,14]$ for a description of such techniques).

It should be emphasized that Laplace formulations can be corrected so as to recover objectivity by adding the missing integral boundary term discussed in Section 7. However, this corrected Laplace formulation is not longer strictly diffusive because, due to the corrective term, the viscous forces do not come exclusively from the Laplace's diffusion operator. Note also that the additional term produces a coupling between velocity components, with this, a computational advantage of Laplace formulations is lost.

The proposed objectivity tests can be used as benchmark tools to check if CFD programs are formulated properly. The numerical experiments presented in Sections 6 and 7, based on annular-cavity flow problems, are equipped with analytical solutions which can be useful to assess correctness and consistency of numerical schemes implemented in open-source codes and commercial software with a minimum of effort.

\section{References}

[1] F. Auteri, N. Parolini, L. Quartapelle, Numerical investigation on the stability of singular driven cavity flow, J. Comput. Phys. 183 (1) (2002) 1-25.

[2] L. Battaglia, J. D’Elía, M. Storti, N. Nigro, Numerical simulation of transient free surfaces flows using a moving mesh technique, J. Appl. Mech. 73 (2006) 10171025 .

[3] J. Blasco, R. Codina, A. Huerta, A fractional-step method for the incompressible Navier-Stokes equations related to a predictor-multicorrector algorithm, Int. J. Numer. Methods Fluid 28 (1998) 1391-1419.

[4] C. Brennen, On the flow in an annulus surrounding a whirling cylinder, J. Fluid Mech. 75 (1976) 173-191. Part 1, March 2006.

[5] A.N. Brooks, T.J.R. Hughes, Streamline upwind/Petrov-Galerkin formulations for convection dominated fows with particular emphasis on the incompressible Navier-Stokes equations, Comput. Methods Appl. Mech. Engrg. 32 (1982) 199-259.

[6] C.H. Bruneau, S. Mazen, The 2D lid-driven cavity problem revisited, Comput. Fluid 35 (3) (2006) 326-348.

[7] CFX. ANSYS, Inc. <http://www.ansys.com/products/cfx.asp>.

[8] W. Chang, F. Giraldo, B. Perot, Analysis of an exact fractional step method, J. Comput. Phys. 180 (2002) 183-199. 
[9] A. Deponti, V. Pennati, L. De Biase, A fully 3D finite volume method for incompressible Navier-Stokes equations, Int. J. Numer. Methods Fluid 52 (2006) 617-638.

[10] J. Donea, A. Huerta, Finite Element Methods for Flow Problems, John Wiley \& Sons Ltd., 2003.

[11] FEATFLOW, University of Dormunt. <http://www.featflow.de/>.

[12] R. Glowinski, G. Guidoboni, T.-W. Pan, Wall-driven incompressible viscous flow in a two-dimensional semi-circular cavity, J. Comput. Phys. 216 (1) (2006) 76-91.

[13] J.L. Guermond, P. Minev, J. Shen, An overview of projection methods for incompressible flows, Comput. Methods Appl. Mech. Engrg. 195 (2006) 60116045.

[14] J.L. Guermond, P. Minev, J. Shen, Error analysis of pressure-correction schemes for the Navier-Stokes equations with open boundary conditions, SIAM J. Numer. Anal. 43 (2005) 6011-6045.

[15] M. Gurtin, An introduction to continuum mechanics, Mathematics in Science and Engineering, vol. 158, Academic Press, 1981.

[16] S.R. Idelsohn, E. Oñate, F. Del Pin, The particle finite element method: a powerful tool to solve incompressible flows with free-surfaces and breaking waves, Int. J. Numer. Methods Engrg. 61 (2004) 964-989.

[17] S.R. Idelsohn, J. Marti, A.C. Limache, E. Oñate, Unified lagrangian formulation for elastic solids and incompressible fluids. Application to fluid-structure interaction problems via the PFEM, Comput. Methods Appl. Mech. Engrg. 197 (19-20) (2008) 1762-1776, doi:10.1016/j.cma.2007.06.004.

[18] S. Kulsri, M. Jaroensutasinee, K. Jaroensutasinee, Simulation of lid cavity flow in rectangular half-circular and beer bucket shapes using quasi-molecular modeling, Trans. Engrg. Comput. Technol. 19 (2007) 401-406.

[19] X. Li, X. Han, An iterative stabilized fractional step algorithm for numerical solution of incompressible N-S equations, Int. J. Numer. Methods Fluid 49 (2005) 395-416.

[20] A.C. Limache, S.R. Idelsohn, Laplace form of Navier-Stokes equations: a safe path or a wrong way? Mecánica Computacional, Asociación Argentina de Mecánica Computacional, AMCA. ENIEF, 2006, pp. 151-168 (XXV).

[21] A.C. Limache, S.R. Idelsohn, R. Rossi, E. Oñate, The violation of objectivity in Laplace formulations of the Navier-Stokes equations, Int. J. Numer. Methods Fluid. 54 (6-8) (2007) 639-664. <http://dx.doi.org/10.1002/fld.1480> .

[22] C.-L. Lin, H. Lee, T. Lee, L. Weber, A level set characteristic Galerking finite element method for free surface flows, Int. J. Numer. Methods Fluid 49 (2005) 521-547.

[23] R. Löhner, C. Yang, E. Oñate, Simulation of flows with violent free surface motion and moving objects using unstructured grids, Int. J. Numer. Methods Fluid 53 (2007) 1315-1338.

[24] M. Lopez, J.E. Hart, F. Marques, S. Kittelman, J. Shen, Instability and mode interactions in a differentially driven rotating cylinder, J. Fluid Mech. 462 (2002) 383-409.

[25] M. Lopez, F. Marques, J. Shen, Complex dynamics in a short annular container with rotating bottom and inner cylinder, J. Fluid Mech. 510 (2004) 327-354.

[26] A. Meseguer, Energy transient growth in the Taylor-Couette problem, Phys. Fluid 14 (5) (2004) 1655-1660.

[27] R.W. Ogden, Non-linear elastic deformations, Series in Mathematics and its Applications, Ellis Horwood Limited, 1984.

[28] OpenFOAM: The Open Source CFD Toolbox, OpenCFD. <http:// www.opencfd.co.uk/openfoam/>.
[29] PETSc-FEM. A general purpose, parallel, multi-physics FEM program. GNU General Public License (GPL). <http://www.cimec.org.ar/petscfem>.

[30] J.P. Pontaza, A new consistent splitting scheme for incompressible NavierStokes flows: a least-squares spectral element implementation, J. Comput. Phys. 225 (2) (2007) 1590-1602.

[31] A. Prosperetti, Numerical simulation of transient free surfaces flows using a moving mesh technique, Phys. Fluid 24 (7) (1981) 1217-1223.

[32] S. Rabier, M. Medale, Computation of free surface flows with a projection FEM in a moving mesh framework, Comput. Methods Appl. Mech. Engrg. 192 (2003) 4703-4721.

[33] R. Schreiber, H.B. Keller, Driven cavity flows by efficient numerical technique, J. Comput. Phys. 49 (1983) 310-333.

[34] R. Tavakoli, R. Babaei, N. Varahram, P. Davami, Numerical simulation of liquid/ gas phase flow during mold filling, Comput. Methods Appl. Mech. Engrg. 196 (2006) 697-713.

[35] G.I. Taylor, Distribution of velocity and temperature between concentric rotating cylinders, Proc. Royal Soc. 151 (1935) 494-512.

[36] G.I. Taylor, Stability of a viscous fluid contained between two rotating cylinders, Phylos. Trans. Royal Soc. A 223 (289) (1923) 1923.

[37] Tdyn/GiD+, CIMNE. <http://www.compassis.com/gidplus/>.

[38] T.E. Tezduyar, J. Liou, D.K. Ganjoo, Incompressible flow computations based on the vorticity-stream function and velocity-pressure formulations, Comput. Struct. 35 (1990) 445-472.

[39] T.E. Tezduyar, S. Mittal, R. Shih, Time-accurate incompressible flow computations with quadrilateral velocity-pressure elements, Comput. Methods Appl. Mech. Engrg. 87 (1991) 363-384.

[40] T.E. Tezduyar, J. Liou, On the downstream boundary conditions for the vorticity-stream function formulation of two-dimensional incompressible flows, Comput. Methods Appl. Mech. Engrg. 85 (1991) 207-217.

[41] T.E. Tezduyar, R. Shih, Numerical experiments on downstream boundary of flow past cylinder, J. Engrg. Mech. 117 (1991) 854-871.

[42] T.E. Tezduyar, S. Mittal, S.E. Ray, R. Shih, Incompressible flow computations with stabilized bilinear and linear equal-order-interpolation velocity-pressure elements, Comput. Methods Appl. Mech. Engrg. 95 (1992) 221-242.

[43] T.E. Tezduyar, Stabilized finite element formulations for incompressible flow computations, Adv. Appl. Mech. 28 (1992) 1-44.

[44] T.E. Tezduyar, Y. Osawa, Finite element stabilization parameters computed from element matrices and vectors, Comput. Methods Appl. Mech. Engrg. 190 (2000) 411-430.

[45] A.J. Wathen, Preconditioning and fast solvers for incompressible flow. Numerical Analysis Group Research Report, Oxford University, NA-04/08, pp. $1-13,2004$

[46] R. Webster, Algebraic multigrid and incompressible fluid flow, Int. J. Numer Methods Fluid 53 (2007) 669-690.

[47] J.C.-F. Wong, M.K.-H. Chan, A consistent splitting scheme for unsteady incompressible viscous flows I Dirichlet boundary condition and applications, Int. J. Numer. Methods Fluid 51 (2006) 385-424.

[48] W. Yue, C.-L. Lin, V. Patel, Numerical simulation of unsteady multidimensional free surface motions by level set method, Int. J. Numer. Methods Fluids 42 (2003) 853-884.

[49] O.C. Zienkiewicz, R.L. Taylor, The Finite Element Method, McGraw-Hill, 1991 vols. I-III. 